\title{
Alterações oculares nos pacientes portadores de lúpus eritematoso sistêmico em acompanhamento ambulatorial
}

\author{
Ophthalmological alterations in outpatients with systemiclupus erythematosus
}

\author{
Tatiana Klejnberg'1 \\ Haroldo Vieira de Moraes Junior ${ }^{2}$
}

\begin{tabular}{|c|}
\hline RESUMO \\
\hline 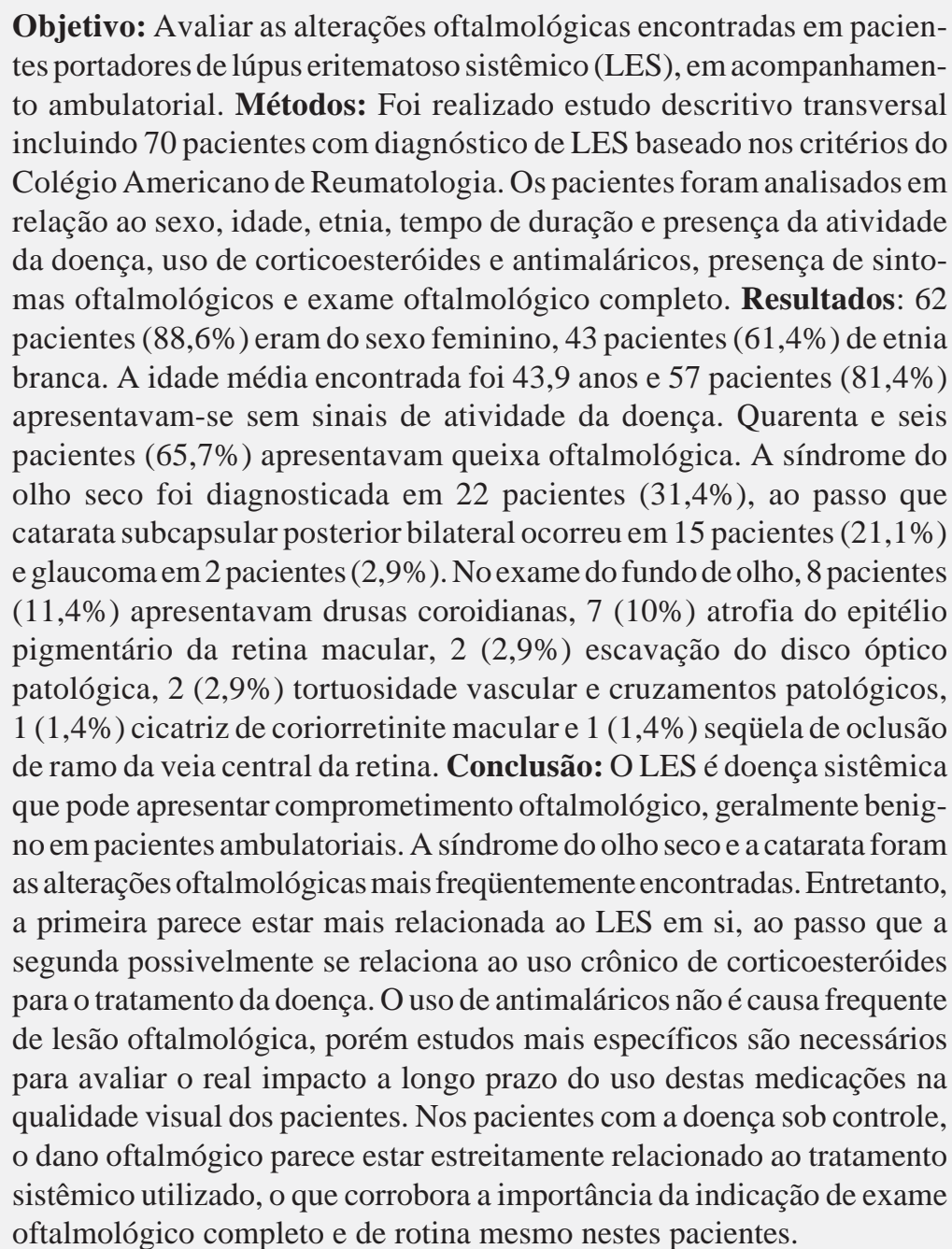 \\
\hline
\end{tabular}

Descritores: Lupus eritematoso sistêmico/complicações; Ceratoconjuntivite seca; Antimaláricos/efeitos adversos; Corticosteróides/efeitos adversos; Catarata/etiologia

\section{INTRODUÇ̃̃̃O}

O lúpus eritematoso sistêmico (LES) é uma doença inflamatória crônica, idiopática, multissistêmica, caracterizada por uma variedade de manifestações clínicas e associada à hiperatividade do sistema imunológico e produ- 
ção de diversos auto-anticorpos. A incidência estimada varia de 1,8 a 20 ou mais casos por 100.000 indivíduos por ano, variando conforme a população estudada. Entre $80 \%$ a $90 \%$ destes pacientes são mulheres em idade fértil, com idade média de $30 \operatorname{anos}^{(1-2)}$. A etiologia do LES não foi ainda completamente esclarecida e vários fatores têm sido propostos para explicar a origem da doença, incluindo genético, hormonal, viral e ambiental ${ }^{(3)}$.

As manifestações clínicas do LES são as mais diversas, podendo se apresentar de diferentes formas e graus de severidade $^{(4)}$. Sintomas sistêmicos não específicos, como febre, fadiga e perda de peso afetam quase todos os pacientes lúpi$\cos ^{(5)}$. O típico exantema "em asa de borboleta" ao longo do nariz e região malar é o achado mais comum. A história natural do LES é muito variável e imprevisível, sendo comum observarmos períodos de remissão e exacerbação da doença. A taxa de sobrevida de pacientes observados na última década aproxima-se de $90 \%{ }^{(6-7)}$.

O LES pode afetar praticamente todas as estruturas oculares e anexos ${ }^{(2)}$, gerando uma grande diversidade de manifestações clínicas, em sua maioria de bom prognóstico, porém podendo haver casos potencialmente graves levando à cegueira irreversível ${ }^{(8)}$.

Além das alterações oftalmológicas induzidas pelo LES, as drogas utilizadas para o tratamento sistêmico da doença são potencialmente danosas para o olho. Os corticoesteróides são reconhecidamente drogas indutoras de catarata e glaucoma, enquanto os antimaláricos podem causar toxicidade retiniana, variando desde alteração pigmentar assintomática e reversível da mácula, até o clássico padrão de maculopatia "em alvo" com perda visual profunda ${ }^{(2)}$.

Este trabalho visa estudar a prevalência das alterações oculares nos pacientes com LES em acompanhamento ambulatorial.

\section{MÉTODOS}

Foi realizado estudo descritivo, transversal, desenhado para avaliar o espectro das manifestações oculares em pacientes portadores de LES em acompanhamento ambulatorial. Foram encaminhados, ao ambulatório de oftalmologia, um total de setenta pacientes de forma aleatória e seriada, entre os meses de maio e outubro de 2003, provenientes do ambulatório do setor de colagenose, com diagnóstico de LES baseado nos critérios do Colégio Americano de Reumatologia ${ }^{(9)}$, sendo adotado como critérios de exclusão presença concomitante de diabetes mellitus, hipertensão arterial severa e síndrome da imunodeficiência adquirida.

Os pacientes foram inclusos no estudo após obtenção do consentimento informado. Os seguintes dados foram considerados: sexo, idade, etnia, tempo de duração da doença, presença de atividade da doença no momento do exame, uso de corticoesteróides e/ou antimalárico durante o curso da doença e presença de sintomas oftalmológicos.

O exame oftalmológico consistiu em medida da acuidade visual com a melhor correção obtida utilizando a tabela de Snellen, teste da tela de Amsler, teste de avaliação do sentido cromático com as pranchas pseudo-isocromáticas de Ishihara, biomicroscopia do segmento anterior, avaliação do TRFL (tempo de ruptura do filme lacrimal), estudo da superfície ocular após coloração pela fluoresceína, teste de Schirmer I, estudo da superfície ocular após coloração pelo colírio de rosa bengala, tonometria de aplanação, biomicroscopia do segmento posterior sob midríase com lente de não contato de 78 dioptrias e oftalmoscopia binocular indireta com lente de 20 dioptrias, executados nesta ordem.

A avaliação do TRFL foi realizada através do uso de uma fita impregnada por fluoresceína, molhada com solução salina não preservada. $O$ ponto de corte de 10 segundos foi utilizado como referência, sendo valores menores que este considerados anormais. Em seguida, foi realizada a avaliação da superfície corneana pela coloração com fluoresceína e a presença de alteração do padrão normal foi registrada. Teste de Schirmer I, foi feito sem utilização de anestésico. Consideramos como normais valores acima de 5 milímetros.

O resultado da coloração com rosa bengala foi graduado de acordo com o método de Van Bijsterveld. Um escore acima de 3,5 por olho, na intensidade da coloração na soma das áreas, foi considerado anormal ${ }^{(10)}$.

Os pacientes classificados como portadores de olho seco foram aqueles que apresentaram resultados anormais em pelo menos três dos testes citados, incluindo-se a queixa relacionada a sintomas de olho seco como um critério diagnóstico.

A pressão intra-ocular, medida através da tonometria de aplanação, foi registrada. Valores até $20 \mathrm{mmHg}$ foram considerados normais.

\section{RESULTADOS}

Dos 70 pacientes incluídos no estudo, $8(11,4 \%)$ eram do sexo masculino e $62(88,6 \%)$ do sexo feminino, $43(61,4 \%)$ pacientes brancos e $27(38,6 \%)$ não brancos.

A idade variou de 14 a 68 anos, com média de 43,9 anos. Os pacientes apresentaram um tempo médio de doença diagnosticada de 12,9 anos, variando de 1,2 a 51 anos.

Com relação à atividade sistêmica da doença, 57 (81,4\%) pacientes apresentavam-se, no momento do exame, sem sinais de atividade, enquanto $13(18,6 \%)$, apresentavam algum sinal de atividade da doença.

Dos 70 pacientes inclusos no estudo, 69 (98,6\%) fizeram uso de corticoterapia e 66 (94,3\%) de antimaláricos em algum momento durante o curso da doença. Dos pacientes que fizeram uso de antimaláricos, nenhum ultrapassou a dose máxima recomendada pela literatura de 4 miligramas por quilograma de peso por dia de cloroquina, ou 6,5 miligramas por quilograma de peso por dia de hidroxicloroquina ${ }^{(11)}$.

Os pacientes assintomáticos do ponto de vista oftalmológico totalizaram 24 (34,3\%). As queixas principais dos 46 pacientes $(65,7 \%)$ sintomáticos estão relacionadas no gráfico 1 . 


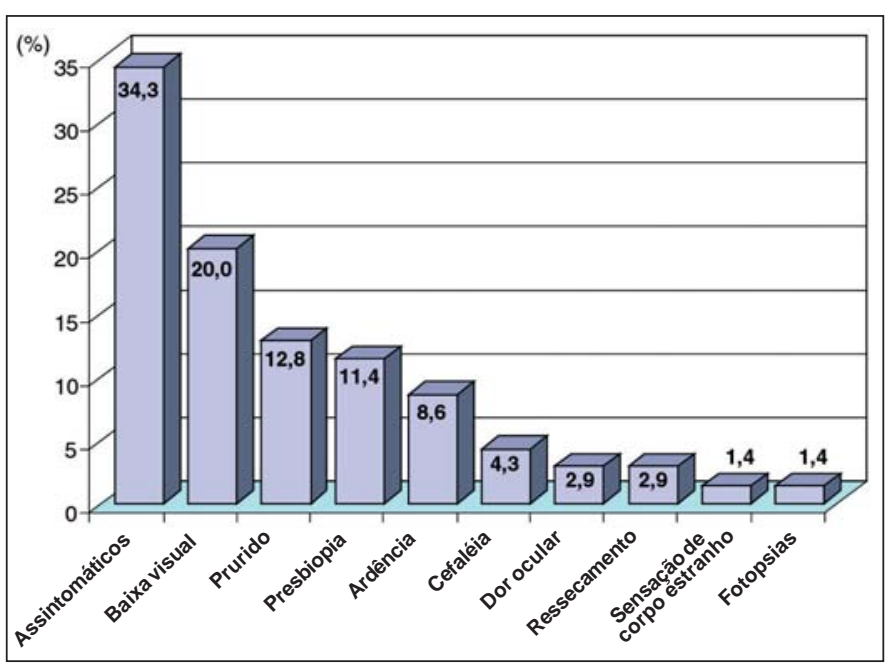

Gráfico 1 - Sintomas oftalmológicos

A avaliação da acuidade visual com correção evidenciou visão de 0,66 , ou melhor, em 57 pacientes $(81,4 \%)$. Treze pacientes $(18,6 \%)$ apresentaram visão menor que 0,66 . Destes 13 , a baixa da acuidade visual foi bilateral em $8(11,4 \%)$ e causada pela catarata em todos estes casos, com exceção de 1 , em que não foi possível determinar a causa da baixa visual. Entre os 5 casos $(7,2 \%)$ unilaterais, 3 apresentavam catarata, 1 cicatriz de coriorretinite macular e 1 atrofia de EPR macular.

Apenas um paciente apresentou alteração do teste feito com a tela de Amsler, sendo esta unilateral. Não foi demonstrada alteração à avaliação do sentido cromático com as pranchas pseudo-isocromáticas de Ishihara em nenhum paciente.

Ao exame biomicroscópico na lâmpada de fenda, foram identificados 29 pacientes $(41,4 \%)$ sem anormalidades. As principais alterações biomicroscópicas encontradas entre os 41 pacientes $(58,6 \%)$ restantes estão listadas na tabela 1 .

$\mathrm{Na}$ avaliação da estabilidade do filme lacrimal, 26 pacientes $(37,1 \%)$ não mostraram alterações, enquanto 44 pacientes $(62,9 \%)$ apresentaram o TRFL anormal.

$\mathrm{O}$ teste de Schirmer I apresentou resultado normal em 53 pacientes $(75,7 \%)$ e anormal em 17 pacientes $(24,3 \%)$. Em relação à coloração pelo rosa bengala, foi encontrado um resultado normal em 47 pacientes $(67,1 \%)$ e anormal em 23 pacientes $(32,9 \%)$.

\section{Tabela 1. Freqüência das alterações biomicroscópicas}

\begin{tabular}{lrr|} 
& $\mathbf{n}$ & $\%$ \\
Catarata & 29 & 41,4 \\
Epiteliopatia ponteada & 16 & 22,9 \\
Pseudofacia & 5 & 7,1 \\
Filamentos/Muco & 6 & 8,6 \\
Pterígio & 4 & 5,7 \\
Blefarite & 1 & 1,4 \\
Entrópio & 1 & 1,4 \\
Madarose & 1 & 1,4 \\
\hline
\end{tabular}

A síndrome do olho seco foi diagnosticada em 22 pacientes $(31,4 \%)$, utilizando-se o critério já descrito anteriormente. Entre os 13 pacientes com a doença em atividade, $4(30,8 \%)$ apresentaram olho seco, enquanto $9(69,2 \%)$ não apresentaram a síndrome. Entre os 57 pacientes sem atividade do LES, diagnosticamos $18(31,6 \%)$ pacientes com olho seco e 39 $(68,4 \%)$ sem a síndrome. A frequiência do número de pacientes com a síndrome do olho seco não variou significativamente de acordo com a atividade da doença ( $\mathrm{p}=1,0$, teste exato de Fisher).

A catarata foi diagnosticada em 29 pacientes $(41,4 \%)$, sendo esta subcapsular posterior bilateral em 15 casos $(21,4 \%)$.

Em relação à pressão intra-ocular, apenas 1 paciente demonstrou valores acima de $20 \mathrm{mmHg}$.

No exame do fundo de olho, não foi identificada qualquer anormalidade em 49 pacientes $(70,0 \%)$. Dentre as alterações encontradas, as drusas coroidianas foram as mais prevalentes, estando presentes em 8 pacientes $(11,4 \%)$. A atrofia de EPR macular foi a segunda alteração mais freqüente, encontrada em 7 pacientes $(10,0 \%)$, ocorrendo bilateralmente em 4 pacientes $(5,7 \%)$ (Tabela 2$)$.

\section{DISCUSS ÃO}

Considerando-se os aspectos epidemiológicos do LES, a predominância do sexo feminino no nosso estudo manteve o padrão apresentado pela doença na literatura mundial.

Estudo em 52 pacientes com diagnóstico de LES reporta uma média de idade de 34 anos ( \pm 11 anos), variando de 16 a 74 anos, com duração média da doença de $3,5 \operatorname{anos}^{(8)}$. Na literatura nacional, foi publicado estudo realizado com 41 pacientes cuja média de idade encontrada foi de 32,22 anos e duração média da doença de 6,17 anos $^{(12)}$.

No nosso trabalho, encontramos uma média de idade de 43,9 anos ( $\pm 10,7$ anos), variando de 14 a 68 anos, com duração média da doença de 12,9 anos, demonstrando uma faixa etária média mais avançada com um maior tempo de duração da doença.

Ao analisarmos a queixa oftalmológica principal dos pacientes, identificamos que entre os 46 sintomáticos, 20 (43,5\%) apresentavam sintomas considerados como relacionados ao quadro de olho seco (entre eles, ardência, dor ocular, prurido, sensação de corpo estranho e ressecamento), demonstrando ser a queixa de olho seco a mais comum entre os pacientes lúpicos.

Em estudo com 60 pacientes lúpicos, foi identificado uma

\section{Tabela 2. Freqüência das alterações fundoscópicas}

\begin{tabular}{lrr} 
& $\mathbf{n}$ & $\%$ \\
Drusas coroidianas & 8 & 11,4 \\
Atrofia EPR macular & 7 & 10,0 \\
Escavação do disco óptico patológica & 2 & 2,9 \\
Tortuosidade vascular e cruzamentos patológicos & 2 & 2,9 \\
Cicatriz de coriorretinite & 1 & 1,4 \\
Oclusão de ramo venoso & 1 & 1,4 \\
\hline
\end{tabular}


prevalência de olho seco de $8,3 \%$, sendo o diagnóstico feito exclusivamente pelo teste com o corante rosa bengala ${ }^{(13)}$. Nguyen et al. definem o olho seco como a manifestação mais comum do LES e estimam sua prevalência em, aproximadamente, $25 \%$, sem fazer referência aos critérios utilizados para o seu diagnóstico ${ }^{(1)}$.

$\mathrm{O}$ artigo de Soo et al. reporta $31 \%$ de pacientes lúpicos com olho seco avaliados apenas através do teste de Schirmer ${ }^{(8)}$.

Os trabalhos citados utilizaram apenas um teste para definir a presença do olho seco ou não mencionam o critério utilizado. A literatura mundial atual reconhece a dificuldade para o diagnóstico desta entidade e reforça que a utilização de vários testes é mais fidedigna já que se trata de doença de natureza múltipla. A seqüência de testes utilizada foi sugerida como apropriada para ser utilizada em uma única visita ${ }^{(14)}$.

No nosso trabalho, os pacientes classificados como portadores de olho seco foram aqueles que apresentaram resultados anormais em pelo menos três dos testes citados. Optamos por utilizar como critério para o diagnóstico do olho seco a anamnese, TRFL, coloração pelo rosa bengala e fluoresceína e ainda o teste de Schirmer, em uma tentativa de aumentar a sensibilidade do diagnóstico. Segundo este critério, foram identificados 22 pacientes $(31,4 \%)$ com diagnóstico de olho seco.

Não foi encontrada relação entre a atividade da doença e a freqüência do olho seco. Isto pode se dever ao pequeno número de pacientes da nossa amostra com a doença em atividade, já que se trata de um grupo em acompanhamento ambulatorial. Outra observação importante é o fato de nossa amostra ser composta predominantemente pela população que tem maior prevalência de síndrome do olho seco, isto é, mulheres na peri-menopausa.

$\mathrm{Na}$ avaliação da acuidade visual dos pacientes, encontramos visão maior ou igual a 0,66 em 57 pacientes $(81,4 \%)$, demonstrando que a grande maioria dos pacientes mantém boa acuidade visual durante o curso da doença. Além disso, a alteração responsável pela baixa visual dos 13 pacientes $(18,6 \%)$ restantes foi, na maioria dos casos, a catarata, evidenciando que o LES em si não é causa comum de baixa visual.

Um paciente apresentou alteração do teste com a tela de Amsler unilateralmente, sendo identificada, no exame do fundo de olho, presença de cicatriz de coriorretinite macular demonstrando que a alteração no teste não está relacionada ao LES.

Yap et al. reportaram uma incidência de $20 \%$ de pacientes com catarata em seu trabalho ${ }^{(15)}$. Em seu artigo, Soo et al. estimam em $14 \%$ o número de pacientes com catarata induzida por corticóide, considerando apenas aquelas bilaterais e subcapsulares posteriores ${ }^{(8)}$.

Em nosso trabalho, temos um total de 29 pacientes $(41,4 \%)$ com catarata, dos quais, $15(21,4 \%)$ apresentavam quadro de catarata subcapsular posterior bilateral. Portanto, podemos notar que a catarata provavelmente induzida pelo corticóide representa $51,7 \%$ do total de pacientes com catarata.

Em um estudo brasileiro, foram avaliados 68 pacientes portadores de LES, tendo encontrado como alterações ocula- res mais significativas o olho seco $(36,7 \%)$, ceratite $(26,4 \%)$, catarata subcapsular posterior $(35,3 \%)$ e alterações do EPR na área macular $(10,3 \%)$, resultados bastante semelhantes aos encontrados por esta autora ${ }^{(16)}$.

Neste presente estudo, apenas 1 paciente apresentou pressão intra-ocular considerada anormal, entretanto um segundo paciente já estava em uso de medicação antiglaucomatosa no momento do exame. Ambos apresentavam escavação de disco óptico de aspecto patológico e fizeram uso de corticoesteróides no curso da doença. Portanto, os 2 pacientes $(2,9 \%)$ foram considerados portadores de glaucoma.

Em uma série de 50 pacientes, encontrou-se 2 casos de pacientes com aumento da pressão intra-ocular e alterações de campo visual ${ }^{(17)}$. Soo et al. relata apenas um paciente com glaucoma na sua série de 52 pacientes ${ }^{(8)}$. Bigolin et al. reportaram 2 pacientes $(4,8 \%)$ com aumento da pressão intra-ocular e escavação do disco óptico consideradas patológicas ${ }^{(12)}$.

Presença de drusas coroidianas foi identificada em 8 pacientes $(11,4 \%)$. Este achado não pode ser relacionado à retinopatia lúpica, mas deve ser levado em consideração, pois pode sinalizar um epitélio pigmentário da retina já comprometido na área macular, que ainda poderá ser exposto à ação de drogas maculotóxicas.

No nosso trabalho não foi identificada presença de retinopatia pelos antimaláricos nos 66 pacientes examinados que fizeram uso de cloroquina ou hidroxicloroquina durante o curso da doença, sendo que todos os pacientes inclusos neste estudo não ultrapassaram as doses máximas diárias recomendadas (250 miligramas por dia de cloroquina e 400 miligramas por dia de hidroxicloroquina), apesar de vários fazerem uso destas por um período maior que 5 anos.

\section{CONCLUSÃO}

O LES é uma doença sistêmica que pode apresentar comprometimento oftalmológico, geralmente benigno em pacientes ambulatoriais.

A síndrome do olho seco e a catarata foram as alterações oftalmológicas mais frequentemente encontradas. Entretanto, a primeira parece estar mais relacionada ao LES em si, enquanto a segunda possivelmente se relaciona ao uso crônico de corticoesteróides para o tratamento da doença. Portanto, para os pacientes com a doença sob controle, o dano oftalmológico parece estar estreitamente relacionado ao tratamento sistêmico utilizado, o que corrobora a importância da indicação de exame oftalmológico completo e de rotina mesmo nestes pacientes.

O estudo sugere que os antimaláricos não são causa comum de lesão oftalmológica, entretanto são necessários estudos mais específicos para avaliar o real impacto em longo prazo do uso destas medicações na qualidade visual dos pacientes.

\section{ABSTRACT}

Purpose: To evaluate the ophthalmological alterations in pa- 
tients with systemic lupus erythematosus (SLE). Methods: A descriptive cross-sectional study was conducted to analyze 70 patients with diagnosis of SLE based on the American College of Rheumatology criteria. Data regarding sex, age, ethnicity, duration of disease, disease activity, use of corticosteroids and antimalarial drugs, and presence of ophthalmological symptoms were recorded and a full ophthalmic examination was carried out. Results: Sixty-two patients (88.6\%) were females, 43 patients (61.4\%) were white. Mean age was 43.9 years, 57 patients $(81.4 \%)$ presented no sign of active disease and 46 patients $(65.7 \%$ ) had no ophthalmological complaints. Dry eye syndrome was diagnosed in 22 patients (31.4\%), bilateral subcapsular cataract in 15 patients (21.1\%) and glaucoma in 2 patients (2.9\%). Fundoscopic evaluation disclosed 8 patients $(11.4 \%)$ with drusen, $7(10.0 \%)$ with RPE atrophy, 2 patients $(2.9 \%)$ presented pathological disc excavation, 2 (2.9\%) vascular tortuosity and arteriovenous crossing changes, 1 patient (1.4\%) had macular chorioretinitis scar and 1 patient (1.4\%) had branch retinal vein occlusion. Conclusion: SLE is a disease that can present with ophthalmic involvement, usually benign in outpatients. Dry eye and cataract are the commonest ocular findings. The former seems to be more related to SLE itself while the latter probably relates to the chronic use of corticosteroids for the disease treatment. Antimalarial drugs do not frequently cause ophthalmic lesions, although further studies must be conducted in order to assess their impact on patients' long-term visual quality. In the patients with the disease under control, ocular damage is more related to systemic treatment what enhances the need for ophthalmological examination as a routine even in these patients.

Keywords: Lupus erythematosus, systemic/complications; Keratoconjunctivitis sicca; Antimalarials/adverse effects; Adrenal cortex hormones/adverse effects; Cataract/etiology

\section{REFERÊNCIAS}

1. Nguyen QD, Foster CS. Systemic lupus erythematosus and the eye. Int Ophthalmol Clin. 1998;38(1):33-60. Review.

2. Arevalo JF, Lowder CY, Muci-Mendoza R. Ocular manifestations of systemic lupus erythematosus. Curr Opin Ophthalmol. 2002;13(6):404-10. Review.

3. Hess EV, Farhey Y. Epidemiology, genetics, etiology, and environment relationships of systemic lupus erythematosus. Curr Opin Rheumatol. 1994;6(5): 474-80. Review.

4. Mills JA. Systemic lupus erythematosus. N Engl J Med. 1994;330(26):1871-9. Review.

5. Iverson GL. Psychopathology associated with systemic lupus erythematosus: a methodological review. Semin Arthritis Rheum. 1993;22(4):242-51. Review.

6. Ginzler EM, Schorn K. Outcome and prognosis in systemic lupus erythematosus. Rheum Dis Clin North Am. 1988;14(1):67-78. Review.

7. Pistiner M, Wallace DJ, Nessim S, Metzger AL, Klinenberg JR. Lupus erythematosus in the 1980s: a survey of 570 patients. Semin Arthritis Rheum. 1991; 21(1):55-64.

8. Soo MP, Chow SK, Tan CT, Nadior N, Yeap SS, Hoh HB. The spectrum of ocular involvement in patients with systemic lupus erythematosus without ocular symptoms. Lupus. 2000;9(7):511-4.

9. Tan EM, Cohen AS, Fries JF, Masi AT, McShane DJ, Rothfield NF, et al. The 1982 revised criteria for the classification of systemic lupus erythematosus. Arthritis Rheum. 1982;25(11):1271-7.

10. van Bijsterveld OP. Diagnostic tests in the Sicca syndrome. Arch Ophthalmol. 1969;82(1):10-4.

11. Marmor MF, Carr RE, Easterbrook M, Farjo AA, Mieler WF. American Academy of Ophthalmology. Recommendations on screening for chloroquine and hydroxychloroquine retinopathy: a report by the American Academy of Ophthalmology. Ophthalmology. 2002;109(7):1377-82.

12. Bigolin S, Oyamaguchi E, Claro C, Bryk Júnior A, Komatsu MCG, Belotto E, Postella E. Achados oculares e fundoscópicos em pacientes com lupus eritematoso sistêmico. Arq Bras Oftalmol. 2000;63(5):383-6.

13. Andonopoulos AP, Skopouli FN, Dimou GS, Drosos AA, Moutsopoulos HM. Sjogren's syndrome in systemic lupus erythematosus. J Rheumatol. 1990;17(2): 201-4.

14. Bron AJ. Diagnosis of dry eye. Surv Ophthalmol. 2001;45(Suppl 2):S221-6. Review.

15. Yap EY, Au Eong KG, Fong KY, Howe HS, Boey ML, Cheah WM, Feng PH. Ophthalmic manifestations in Asian patients with systemic lupus erythematosus. Singapore Med J. 1998;39(12):557-9.

16. Mendes LE, Gonçalves JOR, Costa VP, Belfort Junior R. Alteraçöes oculares no lupus eritematoso sistêmico. Arq Bras Oftalmol. 1998;61(6):713-6.

17. Santos R, Barojas E, Alarcon-Segovia D, Ibanez G. Retinal microangiopathy in systemic lupus erythematosus. Am J Ophthalmol. 1975;80(2):249-52.

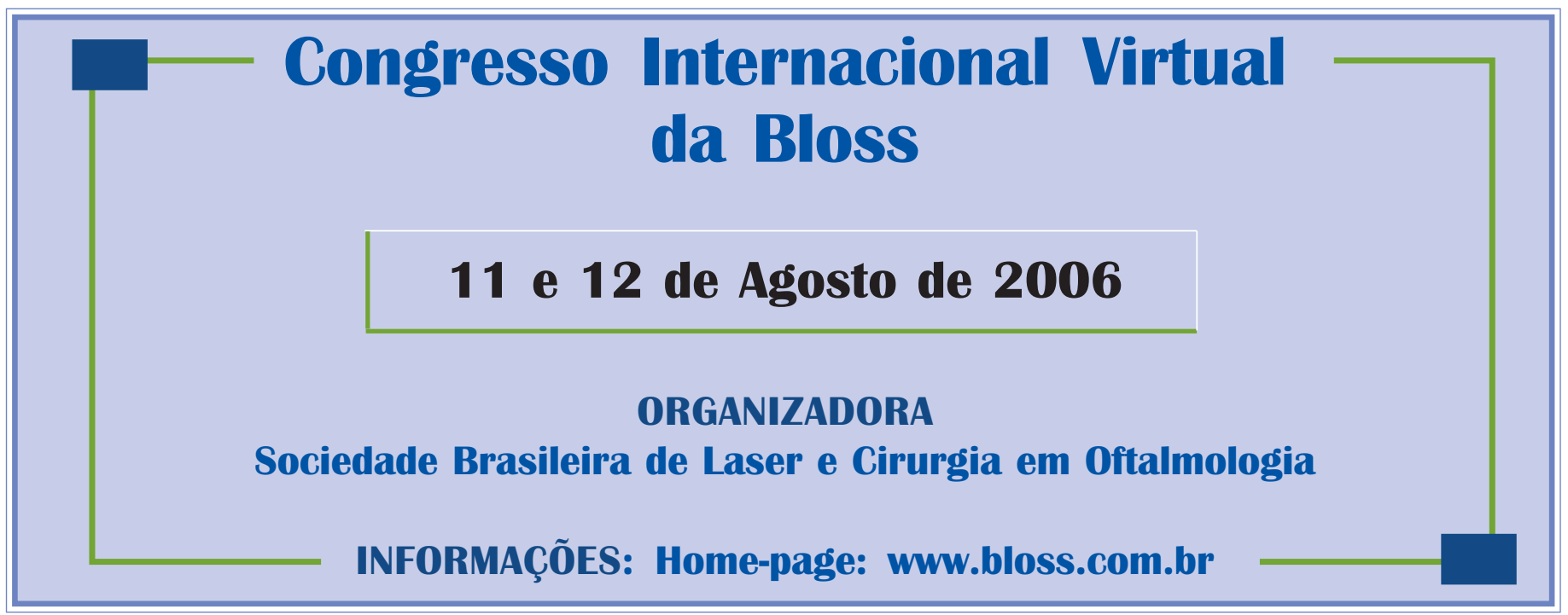

